\title{
Filigrane
}

Écoutes psychothérapiques

\section{De l'Europe aux États-Unis, psychanalyse et consultations parent-nourrisson. Entrevue avec Christine Anzieu-Premmereur}

\section{Eveline Gagnon}

Volume 22, numéro 1, printemps 2013

URI : https://id.erudit.org/iderudit/1017349ar

DOI : https://doi.org/10.7202/1017349ar

Aller au sommaire du numéro

Éditeur(s)

Revue Santé mentale au Québec

ISSN

1192-1412 (imprimé)

1911-4656 (numérique)

Découvrir la revue

Citer ce document

Gagnon, E. (2013). De l'Europe aux États-Unis, psychanalyse et consultations parent-nourrisson. Entrevue avec Christine Anzieu-Premmereur. Filigrane, 22(1), 107-128. https://doi.org/10.7202/1017349ar d'utilisation que vous pouvez consulter en ligne. 


\section{De l'Europe aux États-Unis, psychanalyse et consultations parent-nourrisson. Entrevue avec Christine Anzieu-Premmereur}

\section{Eveline Gagnon}

Invitée à Montréal par la Société canadienne de psychanalyse à l'occasion du congrès annuel de 2012, la psychanalyste Christine Anzieu-Premmereur a accepté d'accorder une entrevue à Eveline Gagnon, pour la revue Filigrane.

Christine Anzieu-Premmereur est psychiatre d'enfants et d'adultes, et psychanalyste membre de la Société psychanalytique de Paris. Depuis août 2000, elle habite et travaille à New York, où elle est professeure assistante en psychiatrie à l'Université de Columbia. Elle enseigne dans le centre de psychanalyse de l'Université de Columbia où elle dirige le département des psychothérapies parents-nourrissons. Membre de la New York Psychoanalytic Society, elle a publié deux livres chez Dunod: Le jeu en psychanalyse d'enfants et Pratiques psychanalytiques avec les bébés. De plus, elle est l'auteure de plusieurs chapitres de livres et d'articles en français et en anglais, sur la fonction maternelle, le développement de l'enfant, le processus de symbolisation chez l'enfant et les techniques d'intervention avec des tout petits.

EVELINE GAGNON : Bonjour Christine Anzieu-Premmereur.

CHRISTINE ANZIEU-PREMMEREUR: Bonjour.

EG : C'est un plaisir d'être avec vous aujourd'hui. Merci d'avoir accepté notre invitation pour cette entrevue pour la revue Filigrane. Vous souhaitez nous entretenir d'un sujet qui vous intéresse particulièrement: le maternel, le rôle des premières relations parent-enfant dans la construction de l'appareil psychique. J'aimerais vous demander pour commencer si vous auriez envie de nous parler de votre parcours. Qu'est-ce qui vous a amenée à réfléchir à cette question du maternel? 
CAP : Il y a eu récemment une invitation de la Société psychanalytique de Paris qui organise tous les ans, avec d'autres sociétés européennes, le Congrès des psychanalystes de langue française, à chaque fois sur un thème particulier avec des rapporteurs sur ce thème. J'ai été choisie avec d'autres collègues pour être rapporteur sur le thème du maternel parce que je travaille en consultation en psychothérapie depuis une vingtaine d'années avec des mères et des nourrissons, des parents, des pères, des mères, parfois des grandsparents, des nannys et de très jeunes enfants. Ça m’a amenée à réfléchir sur la constitution de l'appareil psychique, de l'identité, de la construction du self et du sentiment d'exister, et de la capacité de se reconnaître dans un miroir. Mais aussi, je me suis intéressée à la constitution de la capacité maternelle, aussi bien chez un père que chez une mère, ou chez des adultes qui n'ont pas d'enfant. Comment ça se constitue, comment ça s'organise, comment ça se déforme selon les expériences de la vie et comment ça se transmet? À force de travailler avec des mères en détresse ou dans des désarrois colossaux qui les empêchent d'avoir un bon fonctionnement maternel ou, au contraire, des femmes qui réorganisent leur vie autour de la fonction maternelle, j'ai été amenée à considérer le rôle des parents d'une façon différente de celle que j'avais pu avoir auparavant en travaillant comme psychanalyste d'adultes ou en travaillant comme analyste d'enfants et en voyant les parents comme des objets extérieurs - importants dans la vie de l'enfant, mais externes. Les concevoir comme l'environnement fécondant de l'appareil psychique de l'enfant m'a amenée à travailler différemment avec eux.

EG : Justement, qu'est-ce qui vous a amenée à travailler en psychanalyse d'enfants?

CAP : Ça, c'est loin! J'ai fait des études de psychologie dans les années soixante-dix à Paris, à l'Université de Nanterre. C'est l'époque où, en France, l'influence de Lacan était majeure et celle du structuralisme était très importante. Ce fut pour moi très intéressant, ça m'a permis d'apprendre à lire Freud et la métapsychologie freudienne d'une façon sérieuse et articulée, et j'en ai gardé des traces dans ma formation. Mais c'était une façon de concevoir l'humain que je dirais desséchée, l'humain n'ayant ni corps, ni beaucoup de plaisir sensoriel. Pendant mes études de psychologie, quand je suis arrivée au niveau du doctorat, dans les différents séminaires auxquels j'assistais, j'ai découvert la pensée kleinienne et post-kleinienne. Une seconde influence, évidente, est celle de mon père qui, à cette époque, inventait le moi-peau. La coïncidence de la pensée de Mélanie Klein sur la constitution de l'appareil psychique dans les premières semaines voire les premiers mois de la vie, et 
dans un second temps - ce qui a eu une influence majeure sur moi -, Winnicott pour qui l'appareil psychique se constitue dans un environnement, un bain qui le maintient et lui permet de s'organiser, en plus de l'association avec le corps et une vision de la psychosomatique... Tout ça s'est regroupé autour d'une représentation de l'humain fondée sur quelque chose qu'il ignore, parce que complètement intégré, incorporé ou absorbé en lui. Ce quelque chose qui fait qu'on existe et qu'on transmet à des enfants qu'on va faire ou adopter, à des gens à qui l'on enseigne, à des patients qu'on va soigner... on transmet une partie de cette capacité qu'on a à l'intérieur de soi. Parfois en en étant tout à fait conscient et en la développant, mais le plus souvent, en étant simplement soi-même. Cette capacité de transmettre à d'autres la capacité d'exister avec une existence propre et indépendante, ça semble un peu prétentieux, mais c'est quand même essentiel quand on travaille en psychiatrie de l'enfant.

Après mes études de psychologie, j'ai étudié la médecine pour devenir psychiatre et je me suis orientée très vite vers la psychiatrie de l'enfant. C'était quelque chose d'un peu nouveau à la fin des années soixante-dix en France: la psychiatrie de l'enfant n'était plus seulement asilaire comme elle l'avait été pendant longtemps. Elle commençait à évoluer autour des développements de l'époque sur les dysharmonies et la façon de comprendre tous les déboires de la petite enfance qui donnaient lieu à des déficits: déficits instrumentaux ou troubles graves de la personnalité, troubles du développement comme on dit maintenant. L'énorme obstacle consiste à réparer ce genre de déficits, que je nomme ainsi parce qu'effectivement, ça laisse des traces toute la vie. Il y a des aménagements et du travail psychoéducatif très efficace qui sont accomplis, mais il y a quand même des problématiques qui relèvent sans doute de troubles précoces et d'éléments génétiques, de défaillances précoces vraiment difficiles à réparer afin de donner lieu à un développement harmonieux.

Cela s'est donc avéré pour moi une ligne de pensée et d'intérêt, puis un énorme point d'interrogation. En fait, ce qui m'a animée est la curiosité, l'envie de comprendre des choses qui paraissaient étranges: comment se fait-il que certains enfants ratent la voie d'un développement harmonieux, au moins à certains moments? On peut toujours diagnostiquer des maladies organiques, mais tout le débat sur l'autisme aujourd'hui est absolument significatif de ce genre d'ignorance dans laquelle on est. Qu'est-ce qui s'est passé pour qu'un enfant ait du mal à devenir humain ou bute sur les possibilités de maturation, de développement et de transmission, puis reste bloqué? Non seulement bloqué, mais des pathologies s'organisent, des mécanismes d'un 
registre tout à fait pathologique viennent contrer l'angoisse. Comment cela fonctionne-t-il ? En fait, on n'en sait rien!

La recherche en psychologie, la recherche sur les interactions précoces et la recherche neurologique se sont développées de façon très intéressante, ce qui donne des pistes. Pour l'instant, cela permet de relativiser la toute-puissance de la pensée psychanalytique et de la pensée médicale. La science évolue, mais comment fonctionne un appareil psychique précoce qui donne lieu, à un moment ou à un autre, à un bébé qui arrête de se développer? Souvent les explications génétiques ou neurologiques ont du poids, un impact ; c'est évident qu'il y a des phénomènes de cet ordre, mais quelle est l'interaction de ces facteurs avec les possibilités évolutives et la vitalité d'un enfant, ou avec les événements qui ont lieu dans sa famille à une certaine époque? Il est passionnant de regarder cela en évitant les croyances: sans penser que toutes les mères sont mauvaises - ce qu'on a tendance à faire lorsqu'on s'occupe d'enfants qu'on investit énormément, et qu'on ne fait plus quand on s'occupe de dyades mère-nourrisson. La mère devient dès lors un objet de soin, d'intérêt et de traitement; un être humain avec sa complexité, ses conflits et sa propre histoire. C'est l'emboîtement des deux qui est intéressant.

EG: Vous avez l'impression que cette pratique-là permet d'avoir une vision différente?

CAP : En tout cas, je suis sortie des clivages dans lesquels j'étais personnellement, aussi bien pour des raisons personnelles que pour des raisons d'enseignement, à l'époque. Dans les années soixante-dix en France, la pensée psychanalytique était assez toute-puissante, ce qui était assez agréable quand on prenait cette voie-là, mais qui était quand même une déformation qui consistait à penser que tout venait de l'appareil psychique. On ne pouvait pas avoir la grippe sans se demander quel sens ça avait; sans exclure que ça arrive à des moments où on est un peu immunodéprimé, ça pouvait correspondre à des fluctuations de l'humeur. Mais c'était une pensée radicale qui, à mon avis, a conduit à des erreurs. L'une d'elles a été de considérer que l'appareil psychique des parents était pratiquement toujours responsable du fonctionnement psychique des enfants. C'est quand même une grosse bêtise. Et, surtout, ça mène à des erreurs de traitement. Ça justifie les thérapies familiales : puisque l'environnement est responsable de tout, il faut gérer l'objet-famille. Ce qui peut tout à fait avoir du sens et fonctionne très bien, mais l'appareil psychique de l'individu-enfant qui a besoin de se développer pour lui-même est à ce moment-là négligé ou demeure non soigné comme il pourrait l'être. À l'inverse, ce qui a été poussé à l'extrême par Mélanie Klein est qu'il n'existe 
que l'appareil psychique de l'enfant, qu'on ne traite que cet espace psychique, et que les interactions avec les parents sont négligeables et secondaires. Ou les parents sont responsables de tout, ou ils sont complètement éjectés. Les choses ont beaucoup changé dans les années quatre-vingt, quand toute la psychiatrie du nourrisson s'est développée. Pour citer des noms importants: Serge Lebovici a été le promoteur de ce courant.

EG: Vous avez travaillé avec lui...

CAP : Oui, j'ai travaillé avec lui. Il a été, avec une énergie considérable, celui qui a enseigné, développé, montré comment il travaillait dans les consultations parents-nourrisson. Bertrand Cramer en Suisse, et toute la clinique de psychiatrie de l'enfant qui s'est développée à Genève par la suite avec PalasioEspasa, et qui maintenant continue sur sa trace... c'est un lieu de travail et de recherche très riche. En ce qui concerne mon expérience personnelle, au Centre Alfred-Binet, à Paris dans le $13^{\mathrm{e}}$ arrondissement, nous avons ouvert à la fin des années quatre-vingt et au début des années quatre-vingt-dix une clinique spéciale pour les mères, les parents, et les bébés et les enfants de moins de trois ans. En travaillant avec la vidéo, en revoyant l'enregistrement des consultations qu'on faisait et en ayant un séminaire qui nous permettait de réfléchir ensemble à ce qu'on comprenait de ces séances complexes (puisqu'il s'agissait d'observer un bébé en même temps qu'une mère, un père et les interactions entre les deux parents), et de savoir quand intervenir, avec qui et pourquoi. En parallèle, le même travail était développé au Centre Anna Freud à Londres, où Tessa Barandon a développé un travail parent-nourrisson dans un service public, ce qui permettait à toute une population d'avoir accès à des soins gratuits, et aux psychanalystes et aux psychiatres d'avoir accès à une clinique absolument extraordinaire. Dès qu'on commence à recevoir des nouveau-nés, des enfants de quelques semaines ou de petits enfants qui commencent à marcher qu'on appelle toddlers (c'est-à-dire les enfants de 18-24 mois qui commencent à entrer dans des situations de crise), on apprend une clinique très spécifique à chaque âge et chaque étape de développement. On entrevoit les conflits que cela suscite chez les parents ou la façon dont ceux-ci sont amenés à soutenir ou au contraire à s'opposer à certaines phases de développement de leur enfant. Il y a bien sûr des prédécesseurs: tous les travaux de Margaret Mahler, l'observation de films de bébés suivis pendant les trois premières années de leur vie et toute la dynamique entre la mère et l'enfant. Il y a eu tous les travaux de Selma Fraiberg aux ÉtatsUnis qui consistaient à travailler au domicile de familles particulièrement défavorisées, avec des histoires traumatiques à répétition sur plusieurs 
générations, afin d'interrompre la transmission de phénomènes pathologiques ou traumatiques. On avait un background important.

Et puis, toute la recherche psychologique qui s'est développée dans les années soixante-dix et quatre-vingt, l'observation psychologique de Daniel Stern en particulier et Béatrice Beebe à New York, m’ont appris à regarder de façon très attentive et très précise tout le jeu des modalités d'interaction entre une mère et son bébé: autour du regard, du son, de la musicalité de la voix, du portage, du rythme des qualités de jeu et de la façon dont chacun peut suivre ou pas le rythme de l'autre. À la suite de ces expériences, un système d'empathie un peu particulier se créé, non pas à l'égard de la maman ou de l'enfant, mais à l'égard de la dyade comme une entité en soi, qui permet de suivre tous ses désarrois.

Un autre élément important, typiquement français, est l'Institut de psychosomatique créé à Paris à cette époque. Pierre Marty, de la Société psychanalytique de Paris, a développé une théorie et une pratique auprès de patients atteints de troubles psychosomatiques, en étudiant le fonctionnement psychique qu'il qualifie d'opératoire (dans la concrétude plutôt que dans le fonctionnement symbolique). À ce même endroit, Kreisler (qui était pédiatre) a développé le champ de la clinique psychosomatique du bébé, ce qui a ensuite été repris par Rosine Debray, en même temps qu'à l'Institut de Puériculture de Paris, Michel Soulet développait toute une clinique autour des mères et de leur bébé. J’ai été interpellée par un moment très fécond, dans les années soixante-dix et quatre-vingt, par un champ nouveau qui était celui de la clinique des mères et des bébés.

Ça m’arrangeait bien, parce que j'avais pris la décision contestable de choisir la même profession que mes parents. Mon père était un professeur de psychologie célèbre et un grand psychanalyste qui travaillait avec des adultes borderlines avec des grands troubles du narcissisme. Ma mère était une psychanalyste d'enfants tout à fait reconnue, réputée, et elle enseignait aussi. J'ai eu la chance de trouver une voie spécifique, la clinique du bébé, qui ne les concernait ni l'un ni l'autre. Cela m'a permis de trouver mon identité. Voilà pour les bénéfices secondaires!

EG : Justement, j'aimerais vous poser la question : vous avez nommé quelques personnes, notamment Lebovici, mais qu'est-ce que vous évoqueriez comme votre filiation professionnelle? Les gens marquants? Les rencontres?

CAP : Après avoir dit que les autres étaient mégalomaniaques... Mon grandpère mythique est Winnicott; je m’inscris dans une généalogie imaginaire avec Winnicott. 
EG: Vous n'êtes sûrement pas la seule!

CAP : Oui, j'ai beaucoup de frères et sœurs; on est une grande famille!

Winnicott, donc, pour deux raisons. L'une qui est vraiment très personnelle. Quand j'ai été enceinte et que je me suis coltinée d'avoir des bébés, chose que j'ai adorée - et j'ai eu beaucoup de chance parce que tout s'est bien passé dans ma vie et dans la vie de mes enfants -, ce fut quand même beaucoup de soubresauts émotionnels et d'angoisses à affronter. La lecture de Winnicott - celui qui s'adressait aux mères, aux bébés et aux sages-femmes, avec notamment les émissions de radio, le Winnicott qui parlait à tout le monde - m’a beaucoup interpellée. Et puis bien sûr, j'étais en analyse, ce qui m'a permis d'aller explorer certaines profondeurs. Mais la pensée et la parole de Winnicott éclairaient mes expériences. C'était pour moi fort étonnant de trouver dans des livres écrits très simplement quelque chose qui correspondait à des expériences personnelles que j'avais du mal à mettre en mots; c'était compliqué de se réveiller au milieu de la nuit avec un bébé qui pleure, d'être débordée d'angoisses, et de ne pas pouvoir se rendormir une fois qu'on a calmé le bébé, par exemple. En Winnicott, je trouvais écho et validité à mes expériences, ce qui est génial!

D'autre part, après avoir fait une formation analytique classique (comme on en fait en France), très freudienne bien qu'ouverte sur la pensée kleinienne et celle de Lacan, quelque chose m'a particulièrement intéressée de la pensée de Winnicott: cette notion qu'un enfant fait dans une séance d'analyse ou dans une consultation une expérience nouvelle qui va transformer une partie de son fonctionnement psychique. C'est dire qu'il n'y a pas lieu de trouver la bonne interprétation d'un mouvement inconscient. Il s'agit plutôt d'être dans la pièce avec l'enfant, d'avoir une attention et une qualité de présence qui permettent à l'enfant d'être, d'exister et de réaliser quelque chose, c'est-à-dire de faire un jeu nouveau et une découverte de soi-même. Ça m'a beaucoup facilité la tâche parce que dans ma formation classique, il s'agissait d'avoir une démarche d'attention, d'écoute émotionnelle et analytique des mouvements internes du patient (adulte ou enfant), mais tout passait par le langage. Découvrir avec Winnicott que l'interaction de l'analyste et du patient, surtout si c'est un jeune enfant ou un patient qui régresse, passe par la façon d'être ensemble m'a beaucoup aidée. Ça m'a libérée d'une contrainte un peu intellectuelle ou théorisante, qui consistait à être intelligente en séance: à dire des choses intelligentes aux patients alors qu'il vaut mieux qu'ils les trouvent eux-mêmes, que ça soit intelligent ou pas, mais que ça fonctionne pour eux. À condition de réfléchir à partir du contre-transfert, j'ai ainsi acquis 
une plus grande liberté d'être, une façon d'être avec moi-même afin de donner aux autres la possibilité de trouver une certaine consistance à leur existence. Bien sûr, je ne parle pas de patients névrosés, avec qui c'est rarement à ce niveau-là que l'on travaille. Mais avec de très jeunes enfants dont l'identité n'est pas constituée ou est en train de se dissocier, de se défaire, ou n'est pas assez bien intégrée pour pouvoir se constituer, les expériences de regard ou de jeu sont des moments très vivants dans l'analyse. Je ne prétends pas que ça fonctionne à toutes les séances, mais ça ouvre cette possibilité. La pensée de Winnicott m'a donné une très grande liberté dans la technique analytique, et je constate que lorsqu'on l'applique à bon escient (non pas systématiquement), c'est une façon d'être plus réceptif qu'actif. Winnicott me sert le plus souvent de référence. Tout ce qui concerne l'espace transitionnel, le fonctionnement transitionnel de l'analyste ou l'attention à ce qu'est le fonctionnement transitionnel chez l'autre, m'a donné une marge de manœuvre que je n'avais pas auparavant quand tout passait par le langage et, peut-être $\mathrm{du}$ fait de ma formation de psychologue, par l'explication psychologique.

EG: Vous avez écrit un texte par ailleurs sur l'humour de l'analyste, sur l'analyste qui est quelqu'un qui comprend les blagues...

CAP : Oui, ça c'était la réflexion autour d'un petit garçon; c'est l'héritage Anzieu. Mon père était quelqu'un qui avait un très important et intense sens de l'humour. On n'échappait pas, lors d'un dîner familial, à quelques calembours. Ça faisait absolument partie de la vie quotidienne; il faisait rire les adultes, mais pas toujours les enfants qui parfois en étaient agacés. Quand j'ai grandi, son humour m'est apparu effectivement superbe. L'humour faisait partie de la vie de mon père du fait de l'existence douloureuse qu'il avait eue, avec une mère gravement malade. Je pense que ça l'a tiré d'affaire par rapport à des angoisses psychotiques, des moments de très grande détresse. C'était devenu un style qu'il communiquait aux autres avec un certain brio. Il était aimé pour ça et moi je l'admirais pour ça. Mon propre sens de l'humour est moyen, mais j'aime beaucoup l'humour chez les autres. Voilà : c'est mon système œdipien!

Lorsque mon père est décédé, en 1999, il y a eu des journées d'hommage et l'on m'a demandé de parler à l'occasion d'une de celles-ci. Je me suis trouvée un peu embêtée car je n'avais pas trop envie de jouer à la fille héritière et de traiter d'un sujet psychanalytique. Moi qui ne suis pas une élève de mon père, je me suis dit que la seule chose qui manque dans toutes ces journées scientifiques où l'on rend hommage à Didier Anzieu - il a écrit L'auto-analyse de Freud, Le psychodrame analytique, Les méthodes projectives, Le Moi-peau 
(et tout le monde parle du moi-peau) - c'est l'humour! Du coup, j'ai écrit ce texte. Je n'y avais pas pensé comme un système d'articulation aussi important, mais comme la rencontre entre la pensée et la personnalité de mon père, et le cas de ce petit enfant qu'on qualifierait maintenant d'autiste. Cet enfant a évolué de cette façon très intéressante: lorsqu'il a acquis le langage, assez tard, ça a été tout de suite pour faire des blagues.

Ses blagues étaient des métaphores des terreurs primitives que décrit Winnicott. Il en parlait avec une rigolade extraordinaire. Une partie de ses blagues étaient des blagues d'enfants : si on se met au niveau de l'enfant, elles étaient drôles. Mais quand il a grandi, c'était un sens de l'humour qu'on pouvait tout à fait partager avec lui. C'était un enfant qui avait des mécanismes de repli autistique issus d'une dépression primaire, d'un manque de vitalité, de quelque chose qui s'était cassé dans son développement du fait d'histoires familiales dont il n'y a pas lieu de parler ici. La reconstruction que j'ai faite est que cet enfant a perdu sa vitalité à un moment où l'angoisse de mort a été débordante dans la famille; il a été négligé, non pas par des parents malveillants, mais des parents qui eux-mêmes étaient débordés par d'autres soucis. Il n'avait sans doute pas les ressources internes, génétiques ou physiologiques, pour être plus combatif, et il s'est installé dans une espèce de repli sans autoérotisme ou véritable fonctionnement libidinal. Les séances mère-enfant ont été lourdes et difficiles, mais éventuellement, un espace transitionnel a pu se mettre en place, dans lequel le jeu s'est avéré possible. La mère a pu devenir ludique, on a commencé à construire des toutous en papier, mais c'était encore du registre post-autistique et quand le langage est apparu, ce fut pour rigoler. Ce n'était pas une défense maniaque, c'était vraiment une façon de retourner la détresse en parole. Il y avait sans doute quelque chose de maniaque à pouvoir en rigoler, mais il avait un tel bonheur à construire des phrases et à inventer avec le langage des choses symboliques pour en rigoler. C'est ce cas que j'ai publié. Quand il est revenu quelques années après, il avait parcouru un circuit scolaire normal, mais avait eu quelques déboires à la fin de l'école primaire, ce pourquoi il est revenu avec sa mère en consultation. Celle-ci pensait qu'il ne savait plus du tout qui j'étais mais il a dit: «le psychanalyste, je sais très bien ce que c'est, c'est quelqu'un qui comprend les blagues»!

EG: Alors, ça vient de là.

CAP : Voilà, ça vient de là. C'est un joli cadeau qu'il m'avait fait.

EG: Vous parlez facilement de votre clinique, vous rapportez souvent des cas cliniques dans vos textes, et maintenant vous avez une clinique qui n'est plus 
du tout en France, qui est plutôt aux États-Unis, qu'est-ce que vous pourriez nous dire là-dessus?

CAP : Oui, j'ai déménagé aux États-Unis en l'an 2000 pour des raisons familiales tout à fait externes à un choix professionnel et ça m’a pris quelques années pour pouvoir rétablir mon activité professionnelle à New York. Je travaille beaucoup avec le milieu francophone, mais pas exclusivement, puisque la population de New York est internationale, incluant beaucoup d'Européens, et bien sûr, des patients américains ou anglophones. Ce qui est spécifique à la pratique à New York est d'avoir affaire à des gens qui sont tous des immigrés, parfois des immigrés de luxe, mais quand même des gens déracinés ou qui ont choisi d'immigrer. Ils ont amené leurs enfants à quitter avec eux des origines ou un point de départ quelconque, ou ils ont des enfants qui sont nés en voyage. Cette population a une énergie particulière; ils ont déjà organisé des systèmes de défense assez efficaces à l'égard de ce qui peut être traumatisme, perte, séparation, mais à travers la parentalité, ils vont rejouer avec leurs enfants des conflits à l'égard de leur origine qui vont parfois mettre ceux-ci en difficulté, et parfois non. Cette expérience va constituer une soupape de développement fantastique pour certains enfants, mais pas pour tous. S'il y a de la rancune à l'égard du pays d'origine, ce qui va être transmis aux enfants va être quelque chose de paradoxal: «n'écoute pas tes parents!» Formulé comme cela, ça saute aux yeux, mais en général les parents ne comprennent pas pourquoi les enfants les destituent de leur autorité, alors qu'euxmêmes destituent pourtant leurs propres parents. Ou alors, ce qui est classique dans tous les pays, l'enfant s'adapte de façon remarquable à la langue et au pays d'accueil et c'est lui qui apprend aux parents tout en les désavouant et en les considérant comme plus primitifs et perdus dans le système. Il y a tout un système dépressif et anti-dépressif conflictuel qui se met en place, de même que des rivalités.

Ce que j'observe surtout est la façon de gérer ce qu'on appelle les traumatismes. C'est devenu un mot qu'on utilise pour tous les chocs émotionnels, mais le fait de déménager, de quitter, de se séparer et de le faire sans enjeu transitionnel, entraîne parfois une brutalité dans la façon de gérer la petite enfance. Les parents n'en ont pas conscience bien sûr, sinon ils ne le feraient pas. Il y a tout un « apprentissage » que j'essaie de véhiculer, bien que je ne me sois jamais considérée comme pédagogue ou comme éducatrice. Mais je leur montre comment je gère les séparations à la fin d'une consultation ou d'une séance, ou en mettant l'accent sur la façon dont ils gèrent les transitions avec un enfant qui ne veut pas sortir d'une pièce, qui rouspète après un déménagement ou qui ne 
peut pas supporter une nouvelle école ou une nouvelle langue. Si on le pense en termes de fonctionnement transitionnel, cela ouvre toute une gamme de solutions créatives possibles, plutôt que la radicalité qui souvent est imposée aux familles qui déménagent, qui changent, qui partent.

Et puis à New York, peu de temps après mon arrivée, il y a eu l'attentat du 11 septembre. Cela m'a obligée à réfléchir à toute vitesse et à m’apercevoir que les conceptions que j'avais du traumatisme étaient un peu théoriques et limitées. J’ai été contrainte à réfléchir autrement et à me débrouiller sur place, parce que comme psychanalyste (du moins dans la formation que j'ai eue en France), on a une idée de ce que c'est l'après-coup, c'est-à-dire comment le travail psychique s'est fait ou pas, ou comment quelque chose s'est fossilisé, encrypté dans l'appareil psychique. Mais après un événement traumatique qui vous arrive directement ou qui est arrivé dans la famille, quel processus permet ou pas de l'intégrer? Il y a un processus digestif de l'appareil psychique, une transformation qui s'opère le plus souvent en sexualisant les événements ou alors, qui ne se fait pas. Dans l'après-coup, des années après l'événement, celui-ci a laissé un trou dans l'appareil psychique, ou encore, il y a eu une transformation par laquelle il a pris une couleur éventuellement sexuelle à l'adolescence ou à l'âge adulte. Mais les psychanalystes s'intéressaient rarement au premier coup à cette époque-là. Maintenant, les choses ont changé.

EG : Et vous étiez là...

CAP: J'étais là. J'ai pris le coup sur la tête en même temps que tout le monde, mais par chance, personne autour de moi n'a souffert ou été atteint directement, même si l'événement a été majeur. J'ai été amenée à intervenir avec toute l'équipe de Columbia qui venait juste de m'accueillir, à l'époque. Avec le Parent-Infant Program, nous sommes allés dans les jours suivants accueillir des familles qui venaient chercher leur(s) disparu(s) ou gérer des histoires d'argent. Aussi, tous les immigrés qui tout à coup se retrouvaient sans possibilité de pouvoir garder leur visa, leur logement ou leur travail, ou dans l'impossibilité de mettre leur enfant à l'école. Enfin, des situations tragiques, dramatiques, dans lesquelles se sont retrouvés des enfants, ou ceux-ci étaient laissés dans un coin, le temps de faire toutes les consultations avec les Services sociaux, ou encore, ils étaient eux-mêmes sous le choc de la mort, de la disparition ou des images de l'événement en direct. Certains enfants ont effectivement assisté à la chute des corps à partir des tours ou ils ont vu en boucle les scènes à la télévision; ils ont élaboré le mieux qu'ils ont pu et on a pu faire avec eux quelque chose qui est quand même resté limité... On a improvisé 
comme on le fait dans ces cas-là. Il est vrai que les psychanalystes, les thérapeutes cognitivistes et comportementalistes, avaient une autre façon de faire que les gens qui venaient simplement avec de la bonne volonté. C'est le fait d'être tous ensemble et de relier un peu nos pensées différentes qui nous a permis de constater qu'il y a des choses qu'il ne fallait pas faire, par exemple, qu'il était absolument inutile de retraumatiser tout le monde en les faisant raconter les événements. Là aussi la pensée de Winnicott a été pour moi tellement efficace : afin qu'il y ait possibilité de symbolisation il s'agit, en particulier avec des enfants, d'être là, attentif, puis de recevoir ce qui se passe et essayer d'instaurer un terrain de jeu. Pas forcément un terrain ludique où l'on s'amuse, mais un lieu propice à la représentation. Ça a bien fonctionné dans certains cas, mais pour d'autres, ça n'a pas marché parce que pour moi c'était trop dur. Il y a des choses qu'on a du mal à supporter personnellement puisqu'on était tous sur le coup des événements.

EG: Oui, sur le premier coup.

CAP : Sur le premier coup, absolument. Tout le travail de contre-transfert est en difficulté, comme ce serait le cas pour un psychanalyste qui, venant de perdre un de ses parents, écouterait un patient dans une situation analogue. Il y a tout à coup une espèce de symbiose ou une identification beaucoup trop massive, et une perte de la pensée analytique. Lors des événements de 2001, il y a eu des moments où l'émotion était trop forte. J'ai alors trouvé très intéressant d'observer des gens qui n'étaient pas psychanalystes, des psychologues qui interviennent dans les situations d'urgence et qui avaient des techniques d'intervention. Ces professionnels étaient capables d'installer un cadre. En fait, tout le problème auquel nous nous sommes heurtés à ce moment-là, malgré de très bonnes formations de la pensée, une bonne volonté et une capacité d'attention et d'absorbtion des choses pénibles, est de ne pas avoir pensé au cadre.

EG: Un cadre d'urgence.

CAP: Un cadre d'urgence, un cadre qui contient, qui limite, qui demande aux gens de faire et de ne pas faire, un cadre établi avec une autorité de scout: par exemple, pour organiser des files d'attente pour les gens en deuil, ou autre. C'était remarquable parce que ça a contenu tout le monde. On a retrouvé la valeur du cadre, puis on a retrouvé nos fauteuils d'analyste; à partir du moment où on remet un cadre en place, on peut retrouver une pensée. Winnicott est toujours resté dans l'air...

EG: C'est intéressant parce que vous parlez de vos différentes expériences, de ce qui vous a amenée à vous intéresser et à travailler sur telle ou telle chose 
et puis, c'est un peu votre parcours de vie qui vous a amenée là. Par exemple, votre parcours migratoire qui vous amène à travailler avec des gens immigrants.

CAP: Oui. C'est vrai que l'expérience personnelle... Il se trouve que j'ai déménagé à New York du fait de mon mari; ce n'était pas mon souhait et donc ce fut un énorme effort. Avec des adolescents qui ont du coup une occasion inespérée de faire une crise d'adolescence tonique! Ce furent quelques années un peu rudes. Sur le moment c'est lourd, mais après-coup c'est très intéressant parce que le fait d'avoir traversé cette période, d'y avoir réfléchi, sinon ce n'était pas possible d'analyser ce que c'est que déménager, se retrouver dans un pays anglo-saxon quand on est soi-même latin. Qu'est-ce que c'est que la culture américaine? Quels sont les repères sociaux? Comment la psychanalyse se pratique dans ce pays-là? L'effort intellectuel que ça a demandé a été très fructueux par la suite pour suivre la trajectoire de ceux qui ont des histoires équivalentes, non pas d'autres psychanalystes qui déménagent, mais les habitants de New York. Parfois, ce sont des Américains de souche qui ont un ascendant qui a immigré, ou ce sont des gens arrivés récemment qui ont tout un vécu persécutif, dépressif. C'est extraordinaire la haine de l'étranger que peut entraîner le fait d'être soi-même immigré! Les systèmes projectifs sont très intenses dans le cas où l'on est soi-même un peu attaqué sur ses bases narcissiques. On n'arrive pas dans un pays en roi, on se débrouille. Le fait d'avoir été obligée d'y réfléchir et de faire une tranche d'auto-analyse pour tenir le coup m'a apporté un nouvel éclairage, de même que plus de largeur et d'écoute pour les patients.

Aux États-Unis, j'ai été obligée de prendre en considération dans le travail analytique des modes de pensée assez différents de ceux que j'avais connus : la psychologie du moi, les théories sur le traumatisme comme explication fondatrice des troubles de l'appareil psychique, les systèmes d'intervention beaucoup plus actifs. Je ne dis pas que j'ai changé ma pratique analytique, mais j'ai été obligée de réfléchir à des modes de pensée ou d'intervention différents et, donc, de construire mon identité autour de ça.

EG: Comment vous retrouvez-vous dans ce système qui est quand même très différent de toute votre formation européenne?

CAP: Je suis restée française, il n'y a aucun doute. Mais (j'espère que c'est vrai ce que je vais dire, il n'y a que les autres qui peuvent me dire si c'est vrai) je crois que j'ai vraiment perdu de ma rigidité. J'ai été obligée de dire: «ah bien oui, mais ça pourquoi pas!» C'est intéressant cette critique de l'intellectualisme qui en France est de l'absence de pragmatisme ou, à l'inverse, 
l'aspect très pragmatique et concret des Américains qui me parait parfois manquer de profondeur philosophique. À chaque fois d'être obligée de se dire "oui, mais où suis-je, moi? » Je ne dis pas que c'est vrai ou faux, mais comment puis-je fonctionner? Je suis obligée de retrouver mes racines sinon je ne suis pas solide mais j'ai acquis une beaucoup plus grande liberté par rapport au regard des autres, quant à ce que je fais; ça, c'est à apprendre. J'ai l'âge qu'il faut pour y arriver, mais ça prend du temps.

L'autre chose qui est bien aux États-Unis est que je n'y suis pas la fille de mes parents. Non pas que mes parents ne soient pas connus, mais la France étant un pays paternaliste, on y fait toujours référence aux anciens alors qu'aux États-Unis, les anciens on les tue, quoi! On prend leur place en affirmant «C'est mon tour!» Ça m’a donné une bien plus grande liberté. Je l'expérimente maintenant avec mes étudiants qui apprécient certainement les cours que je leur offre, mais qui ne m'attribuent pas d'autorité spéciale. Cela m'a beaucoup gênée au début parce qu'en France on vénère le professeur même si on l'attaque. Aux États-Unis, il n'y a pas de respect particulier pour les gens qui vous enseignent; il y a au contraire débat. Maintenant, j’y prends plaisir, mais au début j'étais un petit peu dérangée. Je n'avais pas l'habitude qu'on ne me respecte pas d'emblée. Maintenant, j'aime beaucoup le challenge que c'est d'avoir à donner des cours qui doivent toujours intéresser les étudiants, les satisfaire ou même susciter leur curiosité, plutôt que de simplement partager un savoir. Il s'agit d'exercer l'esprit en action plutôt qu'apprendre et savoir des choses. Cela rejoint d'anciens propos français: "la tête bien faite plutôt que la tête bien pleine.» C'est cela que l'on pratique ici.

EG: Vous vous occupez d'une chaire de recherche particulière à l'Université Columbia?

CAP: Ce n'est pas vraiment une chaire de recherche. Dans la faculté de médecine, au sein du département de psychiatrie, il y a un Institut de Psychanalyse. Dans les deux grandes universités de New York, NYU et Columbia, il y a de tels instituts de psychanalyse. On y offre de la formation analytique aux psychiatres et aux docteurs en psychologie, ce qui permet aux psychanalystes d'enseigner et de superviser les résidents en psychiatrie, les internes et les chefs de clinique en psychiatrie. Ils transmettent la conception de la vie psychique et de la méthode thérapeutique analytique à de jeunes praticiens en psychiatrie. Ceci est d'autant plus important que la formation médicale et la formation psychiatrique sont maintenant redevenues purement organiques et chimiques. On ne nomme pas la psychanalyse, d'ailleurs, on préfère le 
terme «psychodynamique» qui est la façon politically correct de nommer la référence à quelque chose qui se passe à l'intérieur, à la vie interne. De la même façon que l'infant mental health, la vie mentale du bébé, est quelque chose qui ne s'explique plus, qu'on peut revendiquer, mais qui a disparu pendant 20 ans. Aucun pédiatre n'est maintenant intéressé à rencontrer un psychologue ou un psychanalyste. L'idée que les enfants aient une vie mentale parait stupide; ils ont un cerveau et il y a des hormones à l'intérieur. On s'occupe de l'ocytocine, on s'occupe de la dopamine, de la sérotonine, des gènes de l'autisme ou de l'homosexualité, ou encore des systèmes complexes de neurones miroirs, et cetera, et cetera. Mais les sentiments, les émotions n'ont aucune importance. Ça revient, toutefois, mais la vie mentale et la vie interne n'existent plus dans la formation des médecins et, pour les psychologues, elles existent, mais comme quelque chose d'observable. On peut toujours s'acharner à observer l'inconscient, mais Freud nous a montré qu'en dehors des lapsus, des mots d'esprit, du sens de l'humour et des rêves, l'inconscient par définition nous échappe, donc ne peut s'observer en laboratoire. Pour certains, il n'a plus de statut puisqu'il n'est pas observable.

Pour revenir à la recherche, celle qui se fait actuellement permet de comparer les traitements psychanalytiques avec les autres traitements. Une recherche qu'on souhaiterait amorcer dans notre département consisterait à évaluer l'évolution d'une dyade, puis le développement de l'enfant à long terme, quand il y a des interventions précoces. Pour revenir à cet institut de psychanalyse dans l'Université Columbia, j'y dirige un département où l'on offre une formation à la psychanalyse de l'enfant, à la psychothérapie de l'enfant, à la psychothérapie parent-nourrisson. C'est une formation d'une durée de deux ou trois ans, avec toute une formation clinique et des cours que j'offre avec des collègues, de même que l'observation de nourrissons à domicile avec supervision sur le mode de ce qui se fait à la Tavistock Clinic de Londres. On forme des psychologues et des psychiatres à être compétents, à évaluer une famille avec un bébé, et à faire des interventions; les interventions sont faites dans des cliniques spécialisées, dans les cliniques d'early intervention aux États-Unis (où l'on évalue les bébés qui ont des troubles) ou dans des services où il y a de la family practice. Donc, en général, dans les milieux défavorisés du Bronx, dans Harlem, il y a des cliniques où mes étudiants sont très bien accueillis parce qu'il y a un besoin énorme de psychothérapies auprès de jeunes enfants et de parents qui ont eu des trajectoires d'immigration ou de traumatisme sur plusieurs générations, qui nécessitent des interventions rapides. Il y a aussi toute une population de mères célibataires, incarcérées, 
ou enceintes et toxicomanes qui mettent leurs enfants dans des situations à risque; dans ces cas, il y a possibilité d'intervention très précoce pendant la grossesse ou avec le bébé par le Parent-Infant Program. Quant à la recherche, on commence à s'y mettre et la développer, mais il a fallu qu'on recrute des gens un peu plus spécialisés que quelqu'un de mon âge formé à la psychanalyse française. Mais nous allons bientôt commencer cette recherche sur les interventions auprès de dyades faites par des psychanalystes, avec une évaluation à long terme des enfants.

EG: Vous parlez de ces cliniques qui permettent d'avoir des interventions précoces avec mères et enfants, que pensez-vous que devient cette intervention, toute la clinique des jeunes enfants?

CAP : Il y a un changement important dans l'envergure internationale qu'a pris ce domaine. Depuis la fin des années soixante-dix, il existe la WAIMH, World Association for Infant Mental Health, qui est l'Association internationale de psychiatrie du nourrisson ou de santé mentale du bébé. Elle rassemble tous les ans des praticiens du monde entier (le dernier congrès était au mois d'avril en Afrique du Sud), aussi bien des chercheurs que des thérapeutes de diverses formations. Il y a des domaines de prévention et de thérapie qui se sont développés dans le monde entier. Mais il y a les aléas de la pensée scientifique, des financements de recherche et des décisions politiques qui font que c'est à la fois quelque chose qui existe, qui a un certain prestige aux yeux de certains universitaires, et qui en même temps, ce que je remarque aux États-Unis, est totalement dévalué par les psychiatres et les pédiatres. Il y a là-bas un refus de reconnaître que la vie psychique précoce nécessite une attention, une évaluation et des interventions préventives, ou des interventions thérapeutiques précoces. J'appelle cela un refus parce que je le défends, mais pour les gens qui ne le défendent pas, c'est simplement l'idée que cette approche est complètement désuète, n'a plus lieu d'être parce que non prouvée scientifiquement. C'est quelque chose qui a complètement disparu. Ce qu'on appelle early intervention consiste à offrir à des enfants qui ont des retards de développement ou d'apparition du langage, de la kinésithérapie, de la psychomotricité et de l'orthophonie. Mais à aucun moment, il ne va y avoir une psychothérapie ou même simplement une rencontre avec un psy de proposée. L'approche dominante consiste en une rééducation partielle autour des symptômes, et à aucun moment l'enfant ne sera pensé en tant qu'un soi qui se développe; parce qu'il ne s'agit pas seulement d'émettre des sons ou d'être capable d'articuler des mots, il s'agit de pouvoir dire «Je» et de pouvoir dire, par exemple, «Je vous emmerde!» C'est quand même l'une des 
premières phrases que les enfants disent sous forme de «non». Mais, de le dire parce que ça sort d'un moi en place, cela est absolument dénié.

Ce n'est pas la même chose en Europe, où une contestation énorme de ce mode de pensée s'amorce, et où surtout, il $\mathrm{y}$ a des choix politiques et financiers qui mettent en péril les interventions préventives et précoces. Aux ÉtatsUnis, c'est quelque chose qui a disparu depuis très longtemps, et une grande partie des critiques portent sur les traitements médicamenteux (parce que les jeunes enfants sont médicamentés aux États-Unis), sur l'absence d'efficacité des traitements comportementaux et des traitements éducatifs des enfants autistes. À l'inverse de l'Europe où notamment la psychanalyse a été totalement interdite en France pour le traitement des enfants autistes et rabrouée dans la plupart des pays, aux États-Unis, il commence à y avoir des considérations autres, à condition de prendre en compte l'aspect organique et de mettre fin à la toute-puissance psychanalytique. De toute façon, la psychosomatique, ce n'est que ça la petite enfance. Il est impossible de considérer un appareil psychique en dehors du corps. L'appareil psychique se construit dans la sensorialité et dans, je dirais, le bon équilibre des viscères. On ne peut considérer l'un sans l'autre. Mais les interventions par le jeu et la parole, le soin accordé aux mères et à leur fonctionnement maternel ont un effet à long terme essentiel. Ça, c'est quand même quelque chose qu'il va falloir revendiquer haut et fort dès maintenant, dans un monde où il faut le démontrer avec la recherche.

EG: Oui, parce qu'on a l'impression justement que c'est toute une approche très pédagogique, éducative des enfants qui est prisée actuellement.

CAP: Oui, sans que les émotions et les affects, surtout les affects, ne soient pris en compte. La psychanalyse apporte la notion d'une conflictualité psychique et que les symptômes de l'enfance peuvent venir d'un moi qui ne se met pas correctement en place, de quelque chose qui ne s'intègre pas, ne se constitue pas et d'une dissociation interne grave pour le développement de l'être humain qui s'organise. Si l'organisation est adéquate, ensuite s'installent néanmoins les conflits et l'angoisse: c'est ça la vie normale. La vie normale est faite de mécanismes de défense, de symptômes. Il y a des symptômes qui vont être invalidants et d'autres qui vont être simplement le signe que quelque chose est en évolution. C'est un mode de pensée très spécifique à la psychanalyse. Si on observe simplement de l'extérieur le fait que tel enfant suce son pouce, fait pipi au lit, mord ses copains à l'école ou refuse d'apprendre à lire et à écrire, on dit: « il va falloir qu'on lui mette un appareil dentaire et de la moutarde sur son pouce. Qu'on lui mette un système électrique qui fait qu’il se réveille la 
nuit pour qu'il arrête de faire pipi. On va lui donner une rééducation orthophonique... » Pourquoi pas? C'est pas mal, mais sans considérer les conflits et les fantasmes sexuels qu'il a autour de l'écriture, je pense effectivement qu'on va en faire un adolescent malheureux. Il ne fera plus pipi, ne sucera plus son pouce et saura écrire, mais il n'aura pas développé des systèmes libidinaux bien organisés, bien en place, pour ensuite avoir un amour de soi, une vie amoureuse et une curiosité scientifique bien placée. Je crois toujours à ce que disait Freud, difficile à croire, mais évident: la réussite d'une analyse ou d'une vie c'est d'être capable d'aimer et de travailler. Cela implique d'avoir de la vitalité intellectuelle et amoureuse, ce qui se perd facilement.

EG: On oublie peut-être le « aimer» dans la formule.

CAP : Oui. Les sentiments sont devenus le résultat des hormones. «Vous n'avez pas assez d'ocytocine, donc vous n'êtes pas capable d'aimer.» C'est sûrement vrai et c'est très intéressant. Toute la recherche sur l'ocytocine m'intéresse énormément. Enfin, j'attends que ça ait abouti de façon à ce qu'on puisse l'utiliser pour observer. Par exemple, une recherche intéressante serait, si l'on pouvait doser l'ocytocine avec juste une petite piqûre sur le bout du doigt et déterminer, après quelques séances d'intervention avec une femme enceinte ou une jeune maman qui présente des troubles du post-partum, si le niveau change. J'aimerais bien savoir si nos interventions et la relation permettent des réorganisations psychosomatiques par lesquelles ces hormones de l'attachement sont influencées, ou pas. On n'en sait rien! On sait qu'avec le manque d'ocytocine, il n'y a pas de tendresse, pas trop d'intérêt pour les enfants. Est-ce que ça se répare par les interventions sur la vie affective? Ça m'intéresserait beaucoup.

EG: Vous parlez de toute cette complexité dans l'intervention précoce, à la fois l'environnement, à la fois la vie psychique de l'enfant, à la fois tous les éléments organiques et génétiques. J'aime beaucoup vos écrits parce que, entre autres, vous parlez souvent d'exemples cliniques. Je ne sais pas s'il y a un exemple clinique qui vous viendrait par rapport à vos intérêts pour toute la question du lien parent-enfant?

CAP : Ce qui m'a intéressée ces dernières années est le fonctionnement maternel. Je vais vous donner un exemple qui est très simple, et qui a été filmé. Il s'agit d'une consultation au Centre Alfred-Binet. Les patients acceptaient que la première consultation soit filmée sachant qu'on n'utiliserait ces vidéos que pour la recherche; ce qu'on a fait. C'est en revoyant la consultation que j'ai découvert quelque chose, parce que sur le moment j'étais dans l'action. Il s'agissait d'une mère avec son bébé de trois mois et demi qui venait d'être 
mis à la crèche parce que la mère reprenait son travail. Cette maman arrive dans un état de désarroi majeur, parce qu'elle ressent que son enfant s'est déconnectée. C'est son premier enfant, une petite fille. Quand elle va la chercher le soir à la crèche, elle n'a plus de contact avec son bébé. Elle est dans la crainte de l'autisme, et pense que son enfant a changé et s'est coupée d'elle. C'est une très jolie consultation avec une mère et son bébé qu'elle a installée face à moi, face à la caméra, parce qu'elle vient me voir en tant que spécialiste qui va lui dire si son bébé est autiste ou pas.

EG: Elle vous le montre.

CAP : Elle me le montre, et le bébé ne peut pas voir sa mère. Il sent le dos de sa mère, ses mains, mais il est directement placé face à moi. Le bébé va s'écrouler, se mettre à glisser sur les genoux de la mère et se mettre dans un état de dépression... du moins, le sentiment que cela donne à un adulte construit est la dépression. Cet enfant se vide complètement après un moment d'angoisse à force d'être comme ça, flanqué devant moi. Or, cette mère ne prend pas conscience du malaise de son enfant parce qu'elle plongée dans sa propre angoisse et dans l'attente de me parler. Elle est très accrochée à mon regard même si je regarde le bébé et lui parle régulièrement. Elle dit alors «oui, oui, je la remonte», puis elle repart. Elle a un besoin narcissique, tout à fait justifié, très intense, de communiquer avec moi et le bébé retombe dans son grand malaise. Il se trouve que, par chance, le bébé a faim. C'est l'heure de la tétée. Il commence à rouspéter. C'est un bébé très chouette et quand même bien construit. Il se met à sucer ses lèvres, à avoir toute une pratique buccale qui manifeste une capacité d'attente malgré tout cet état de désarroi. Et puis, à un certain moment, il manifeste vocalement qu'il va falloir faire quelque chose. La mère sort alors son biberon et, devant la caméra et devant moi, elle installe son bébé. Il y a un premier échange de regard, elle lui donne le biberon et, hop, recommence à me regarder et me parler en donnant le biberon. Le bébé tète, mais manifestement - on voit le réflexe de Moro : les bras s'écarter - dans un état de tension et de malaise. J'interromps la mère; ça fait un moment qu'on parle ensemble. Je lui dis: «Écoutez, je crois maintenant qu'on a bien parlé de beaucoup de choses, mais moi je viens de remarquer que votre bébé attend que vous la regardiez.» Cette mère va découvrir et regarder son bébé qui immédiatement accroche son regard; la mère lui fait un grand sourire. Le bébé se met à téter et son bras se remet en place, se met à toucher le foulard de la mère, leurs deux doigts s'accrochent et, ça y est, il y a une espèce de bulle. Et surtout, la mère se redresse et tient son bébé normalement: le bébé qui avait les jambes écartées qui partaient 
dans le vide se trouve à les regrouper. L'échange de regard est d'excellente qualité. À ce moment, la mère dit «Je ne savais pas que ma fille avait besoin de moi.» Puis elle ajoute: «ah, mais alors... je suis mère!»

Ce dont nous avions parlé jusqu'à présent était de sa mère à elle, déprimée, en hôpital psychiatrique, la laissant toute seule à la maison. Elle s'était développée comme une petite fille hypermature, s'était toujours débrouillée seule. Mais elle avait des clivages qu'elle pouvait très bien décrire; il y avait chez elle des moments persécutifs. Du reste, très jeune, sa vie était indépendante et organisée, et elle avait le sentiment qu'elle était en train de reproduire ça avec son bébé: son bébé s'était déjà détachée d'elle, la quittait et allait gérer sa vie toute seule. Elle savait que pour un bébé de trois mois et demi ce n'était pas possible, que ça allait être une douleur intolérable. Au moment où je lui dis qu'elle est mère - alors que le transfert positif était massif, puisque j'étais la personne qui allait tout dire ou tout guérir - au moment où elle est renommée comme mère, elle va se mettre à fonctionner, et le bébé va se réorganiser et se réintégrer. Dans la suite de la consultation, un certain nombre de choses se sont développées, mais rien de nouveau par rapport à ce momentlà qui a vraiment articulé quelque chose. À la fin de cette consultation, la petite fille a fini son biberon, la mère la remet là et la petite fille a un sourire absolument superbe. Elle me regarde et elle se met à babiller. Je lui dis: «ça fait du bien. On s'appuie sur sa mère.» C'est vrai qu'elle appuie son dos; il y a quelque chose de ça. Le lendemain, cette femme m'a téléphoné pour me dire: « vous avez dit quelque chose à la fin de la consultation... je ne me souviens pas très bien. Vous avez vraiment dit qu'on peut s'appuyer sur une mère?» À partir de là, nous avons eu des rencontres intéressantes. C'est un exemple du système d'emboîtement qui s'installe avec un thérapeute qui a une fonction maternelle, même s'il ne s'agit pas de materner la mère; il s'agit d'être là, de ne pas prendre sa place, de la remettre dans sa situation de mère. EG: C'est de porter la mère...

CAP: Oui. À ce moment-là, la mère peut porter le bébé. C’est un exemple d'une situation très simple, mais ça se joue dans des situations plus complexes où il peut y avoir un important niveau d'angoisse. Par exemple lorsque le bébé suscite l'angoisse parce qu'il est malade, ne mange pas, ne dort pas, ou encore, lorsqu'un bébé est prématuré, présente un trouble congénital ou nécessite une opération chirurgicale. Ça rend les parents fous. Il y a classiquement les deuils ou les événements traumatiques qui arrivent pendant la grossesse ou peu de temps après la naissance d'un bébé, qui font que la mère est complètement distordue dans sa fonction maternelle. Je ne dis pas qu'on fait des interventions 
miraculeuses; c'est parfois simple et parfois long. Même s'il s'agit parfois de quelques interventions seulement, l'important est d'être bien là, présent, afin de redonner à la mère sa capacité de transformer les angoisses et d'être à nouveau compétente, présente et attentive à son bébé. Ce sont là des exemples simples; tout le monde le fait dans ce métier-là. C'est ce que j'ai appris à l'époque avec Serge Lebovici et tous les gens qui faisaient cela en France. Il y a maintenant les interventions plus spécifiques autour d'enfants dont on pense qu'ils ont précocement quelque chose qui ne va pas dans le contact, tout ce qu'on appelle le spectre de l'autisme. C'est le mot qu'on utilise aujourd'hui pour toute une gamme de symptômes qu'on ne sait pas comment nommer ou expliquer. Mais ce n'est pas forcément la peine de nommer, parce que ça se transforme si on intervient. À mon avis, si on l'appelle autisme, on fait des dégâts en donnant le nom d'un déficit pour lequel il n'y a pas de traitement. On rend les parents malades sans raison, comme si l'on disait «votre enfant a une trisomie». C'est pas du tout la même chose un bébé qui se replie, se coupe, et interrompt momentanément son développement, non pas à cause d'un déficit organique, mais en raison d'un épisode dépressif, même si ce n'est pas toujours le cas, ça l'est bien souvent. Si l'on peut redonner à la mère une capacité de contenance et une certaine vitalité à l'enfant, le développement redémarre. Je ne dis pas que ça marche tout le temps ni que l'autisme, ça n'existe pas, mais je dis qu'on diagnostique à tort l'autisme lors d'événements qui sont des mouvements de régression ou d'arrêt dans le développement, mais qui ont une autre valeur qu'une valeur autistique. Si on les nomme comme ça, on entraîne un désinvestissement de cet enfant par les parents. Ça cause beaucoup plus de dégâts et c'est plus difficile que si on voit un bébé dont les parents disent «Oh mon Dieu, je suis très angoissé, je ne sais pas ce qui lui arrive!», sans pour autant donner un diagnostic.

EG: Vous nommez cela un travail de redémarrage.

CAP : Oui, de redémarrage. En espérant que ce soit possible. Il y a toujours de la vitalité à trouver quelque part, mais il y a des limites à ce genre d'intervention. Le petit garçon qui a le sens de l'humour, c'est vrai qu'il a parlé très tard. Aux États-Unis, tous les enfants qui ont des troubles du développement précoce sont qualifiés d'autistes. C'est effrayant, mais ça l'est un peu moins maintenant parce que c'est devenu banal. Par exemple, un petit enfant qui avait été qualifié d'autiste, il avait 20-24 mois et était effectivement assez inerte...

EG: C'est très tôt...

CAP: Je vois facilement des enfants entre un et deux ans qui ont des troubles du développement avec des interventions mère-enfant ou père-mère-enfant. 
On essaie toujours d'avoir le papa une fois de temps en temps. Je travaille le samedi exprès pour voir les papas. Au moins une séance par mois avec le papa qui est là, ou le père seul pour pouvoir lui parler de ce qui se passe avec son enfant. Ce sont des séances où on essaye de jouer. Dans cet exemple, le petit enfant était très replié, inerte, triste, vide. Le travail a très bien fonctionné avec une maman pleine de bonne volonté, mais cet enfant-là est resté avec un symptôme: il retenait ses selles... symptôme post-autistique, de garder à l'intérieur et d'érotisation sadique-anale très très puissante. C'est un enfant qui a eu des troubles de la défécation jusqu'à l'âge de 7 ans. Il faut se dire que c'est formidable par rapport au petit enfant qu'il était à l'âge de deux ans : il parle, il va à l'école, il est vivant, il joue, il a des copains. C'était un changement colossal, mais il reste un symptôme qui correspond à un moment de désorganisation de la libido. Quand elle se remet en place, des traces des systèmes de contrôle interne très rigides persistent. C'est un exemple parmi d'autres. Il y a des enfants qui parlent très tard avec tous les troubles d'accès à la symbolisation que ça représente. Je pense que ce sont des interventions nécessaires: la plupart du temps, on fait de la prévention, mais ce n'est pas bien reconnu et il va falloir qu'on s'attèle pour le démontrer.

EG: On sent bien tout un mouvement inverse.

CAP : Oui. Ça serait bien que les pédiatres puissent à nouveau avoir accès à ce genre de travail en commun. Lorsqu'on parle avec les pédiatres qui ont quand même la charge très lourde de la santé d'un enfant, il faudrait leur montrer qu'on est en parallèle avec eux, qu'on n'est pas des compétiteurs. On peut travailler conjointement. Parfois, c'est tellement enrichissant pour tout le monde. Pour eux, je pense que ça leur apprend des choses sur la dynamique mère-enfant. Nous ça nous apprend toute la valeur du travail minutieux du pédiatre. Je reviens à Winnicott: il était quand même pédiatre. Il y a une façon de faire attention à la santé physique et à l'utilisation du corps; c'est quand même ça la santé mentale, d'être bien dans son assiette. Si on n'a pas un corps solide et jouissif, source de plaisir, il manque quelque chose d'essentiel. De même pour tout ce qui est de la curiosité scientifique, la curiosité épistémologique qui nous mène, sinon on s'ennuie dans la vie, c'est mortel! Et éventuellement, on fait un ulcère d'estomac, c'est dommage!

EG: Madame Premmereur, je vous remercie infiniment pour cet entretien et votre grande générosité. Merci en mon nom et au nom de la revue Filigrane.

CAP: Je vous remercie pour votre attention. Je sais ce que c'est de faire attention, et j'apprécie beaucoup. Merci ! 